\title{
, Hybrid-ARQ-Aided Short Fountain Codes Designed for Block-Fading Channels
}

\author{
Hong Chen, Robert G. Maunder, Member, IEEE, Yi Ma, Senior Member, IEEE, \\ Rahim Tafazolli, Senior Member, IEEE, and Lajos Hanzo, Fellow, IEEE
}

\begin{abstract}
5 Abstract-As a benefit of their inherent rateless nature, fountain 6 codes constitute a favorable choice for protecting packet-based 7 transmissions in the physical layer for wireless channels having 8 varying quality. However, previous research has revealed that the 9 performance of fountain codes substantially degrades as their 10 block length is reduced. Three structural phenomena of the 11 Tanner graph were identified by Mackay in the hard decoding 12 of fountain codes on binary erasure channels (BECs), which may 13 be referred to as having "no degree-one check nodes (CNs)," 14 "no emerging degree-one CNs," and "uncovered variable nodes 15 (VNs)." In this paper, we explicitly analyzed how these structural 16 phenomena influence their soft decoding algorithm. Furthermore, 17 these phenomena are shown to be responsible for the high error 18 floors when fountain codes are transmitted over noisy fading 19 channels, particularly for the transmissions of short blocks. To 20 eliminate the influence of these structural phenomena, we con21 ceived a technique of generating a few specifically encoded bits 22 with the aid of the associated Tanner graph. Simulation results 23 have demonstrated that our improved Raptor (IRaptor) codes 24 significantly reduce the packet loss ratio (PLR) of conventional 25 fountain codes, despite imposing reduced low complexity. Finally, 26 we conceive a novel adaptive hybrid automatic repeat request 27 (HARQ) scheme based on a lookup table (LUT)-aided technique, 28 which may adapt its coding rate for each transmission. Our 29 simulation results demonstrated that the proposed IRaptor HARQ

30 achieves a similar performance to the Long-Term Evolution (LTE)

31 turbo-coded HARQ scheme or even outperforms the LTE arrange-

32 ment for block length in excess of $\mathbf{1 0 0 0}$ bits.
\end{abstract}

33 Index Terms-Author, please supply index terms/keywords for 38 your paper. To download the IEEE Taxonomy go to http://www. ieee.org/documents/taxonomy_v101.pdf.

\section{INTRODUCTION}

38 T $\mathrm{N}$ recent years, fountain codes [1]-[3] have attracted sub39 stantial attention since they are capable of approaching the 40 capacity of binary erasure channels (BECs). Hence, most pub41 lications focus on protecting the transmission of packets over

Manuscript received January 24, 2013; revised October 18, 2014 and December 20, 2014; accepted January 2, 2015. The work of L. Hanzo was supported by the European Research Council under its Advanced Fellow Grant. The work of H. Chen was supported by the National Natural Science Foundation of China under Grant 61401067. The review of this paper was coordinated by $\mathrm{C}$. Ibars.

H. Chen, Y. Ma, and R. Tafazolli are with the University of Electronic Science and Technology, Chengdu 610051, China, and also with the Centre for Communication Systems Research (CCSR), University of Surrey, GU2 7XH Surrey, U.K.

R. G. Maunder and L. Hanzo are with the School of Electronics and Computer Science, University of Southampton, SO17 1BJ Southampton, U.K.

Digital Object Identifier 10.1109/TVT.2015.2388632
BECs. Additionally, their rateless property facilitates code-rate 42 adaptation based on the near-instantaneous channel quality. 43

The rateless property is capable of achieving the highest pos- 44 sible throughput, while approaching a potentially zero outage 45 probability. Therefore, Erez et al., [4] studied the construction 46 of rateless codes based on traditional fixed-rate codes originally 47 conceived for Gaussian channels. To obtain the rateless prop- 48 erty, Chen et al., [5] proposed a Raptor-like method, which may 49 extend a low-density parity-check (LDPC) precode from an ini- 50 tial coding rate to any arbitrary rates. Due to the inherent rate- 51 less feature of fountain codes, in [6]-[12], the performance of 52 fountain codes employed as a channel code in the physical layer 53 for transmission over noisy fading channels was studied. More 54 specifically, Palanki and Yedidia [6] revealed that Luby trans- 55 form (LT) codes suffer from error floors, whereas Raptor codes 56 [7] may achieve a lower bit error ratio (BER) than that of LT 57 codes. However, Kasai and Sakaniwa [12] indicated that Raptor 58 codes also exhibit high error floors and designed their outer code 59 as a multiplicative repetition code, while adopting a nonbinary 60 LDPC inner code for improving the performance. Etesami and 61 Shokrollahi [8] derived the necessary conditions of arriving 62 at an optimal degree distribution for transmission over binary 63 memoryless symmetric channels. Furthermore, Cheng et al., 64 [11] demonstrated that Raptor codes may fail to achieve ca- 65 pacity, when the channel's SNR lies outside a specific SNR 66 interval.

However, the performance of fountain codes over noisy 68 channels is not as appealing as anticipated by the given studies 69 under idealized simplifying assumptions, not even at high 70 block lengths. For example, LT codes and Raptor codes have 71 high error floors, as shown in [6] and [12], whereas in [7], the 72 discrepancy between the average rate of Raptor codes and the 73 channel capacity was quantified. Moreover, the performance 74 degrades dramatically, when fountain codes are invoked for 75 protecting short blocks. As our new contribution, we reveal 76 that the modest performance of fountain codes is not only due 77 to having undesirable channel conditions but owing to having 78 a suboptimum Tanner graph structure as well. Tanner graphs 79 [13] efficiently visualize the encoding and decoding process 80 of fountain codes. We analyze how a set of three structural 81 phenomena of the Tanner graph as identified by Mackay 82 in the hard decoding of fountain codes [1] degrades their 83 soft decision. These phenomena are: having "no degree-one 84 check nodes (CNs)," "no emerging degree-one CNs," and the 85 presence of "uncovered variable nodes (VNs)," which again 86 degrade the achievable performance of their soft decoding. 87 
88 We show that the occurrence of these phenomena is mitigated 89 although not entirely eliminated - by the family of systematic 90 fountain codes [14] and by the degree distributions relying on 91 high-degree fractions [1], [3]. Following this, when aiming for 92 transmitting short blocks, we conceive improved Raptor (IRap93 tor) codes by employing our new technique of transmitting 94 several specifically encoded bits, which are carefully generated 95 for eliminating these three structural phenomena of the Tanner 96 graph. A simple degree distribution may be adopted with the aid 97 of these specifically encoded bits. As a result, the SNR required 98 at a given packet loss ratio (PLR) reduced by more than $1.5 \mathrm{~dB}$, 99 despite the fact that the decoding complexity is halved 100 compared with that of conventional systematic fountain codes. 101 Furthermore, we combine IRaptor codes with hybrid auto102 matic repeat request (HARQ) in order to construct an adaptive 103 HARQ scheme. In our proposed IRaptor-coded HARQ scheme, 104 the transmitter is informed about the channel quality by the 105 channel quality indicator (CQI) messages fed back from the 106 receiver. As a benefit, it becomes capable of chasing the appro107 priate coding rate, regardless of the specific channel conditions. 108 It is also capable of passing/delaying its transmissions, if the 109 channel is deemed to be in deep fade.

110 In [12], the "high-error-floor problem" of conventional foun111 tains codes, which was achieved by using a repetition code as 112 their inner code and adopting a nonbinary LDPC outer code, 113 has been carefully addressed. Both the multiplicative repeti114 tion code and the nonbinary LDPC code rely on operations 115 over $\operatorname{GF}\left(2^{m}\right)(m>1)$, instead of the simpler exclusive-OR 116 (XOR)-based addition of bits as in conventional Raptor codes. 117 Compared with our IRaptor codes, it transpires that this im118 plies having a significantly higher encoding and decoding 119 complexity. Moreover, the multiplicative repetition inner code 120 introduced in [12] actually operates as an LT-like code in 121 conjunction with a specific degree distribution, which cannot 122 guarantee circumventing any of the aforementioned three struc123 tural phenomena of the corresponding Tanner graph. Based 124 on the analysis of Section II-B, we infer that these structural phe125 nomena may inject decoding errors into the outer LDPC code, 126 which may exceed its correction capability. Therefore, we may 127 reasonably hypothesize that our IRaptor codes compare well 128 with the multiplicatively repeated nonbinary LDPC codes pro129 posed in [12]. These issues may be explored in our future work. 130 Chen et al., [5] attempted to propagate the rateless property 131 to the class of LDPC codes. To reduce the coding rate, they used 132 LT-like encoding methods for generating more VNs based on a 133 LDPC precoder having an initial coding rate. More specifically, 134 a conventional LDPC precoder first generates an appropriate 135 number of VNs for the sake of arriving at an initial coding 136 rate based on an optimized protograph. This protograph is then 137 optimally extended each time, when a new VN and a new $\mathrm{CN}$ 138 are added into it, until a final coding rate is reached. When 139 comparing it to our IRaptor codes, this Raptor-like LDPC code 140 has a more complex encoding process since finding the optimal 141 protograph will be repeated many times while arriving from the 142 initial coding rate to the required coding rate. Furthermore, the 143 final extended protograph represents the LDPC code's parity144 check matrix, which has to be transformed to the generator 145 matrix for encoding, hence, further increasing the encoding

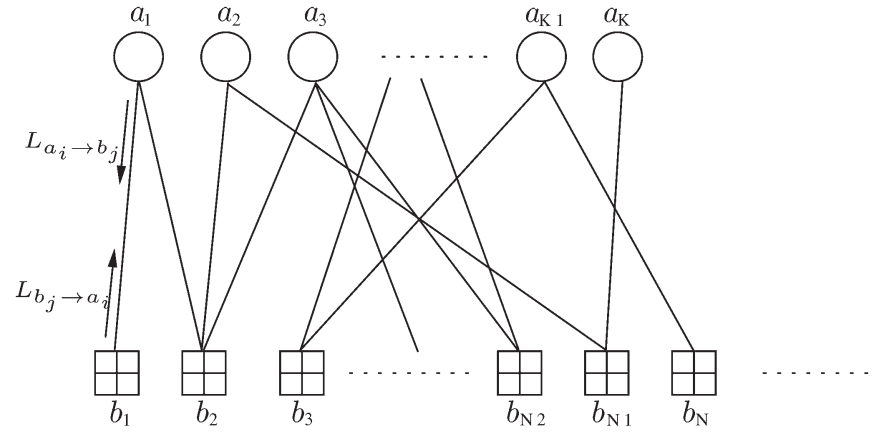

Fig. 1. Tanner graph representing fountain codes, where the hollow circles denote source bits. Each square represents an encoded bit generated by the bitwise XOR sum of its connected source bits.

complexity as well. We may present a quantitative performance 146 comparison between the family of Raptor-like LDPC codes and 147 our IRaptor codes in our future research.

148

The organization of this paper is as follows. In Section II, 149 we briefly introduce soft fountain codes and analyze the cor- 150 responding Tanner graph. A novel method is proposed in 151 Section III for improving the performance for the transmissions 152 of finite-length blocks. Our simulation results are presented in 153 Section III-B for characterizing our IRaptor codes in contrast 154 to both conventional fountain codes and to the Long-Term 155 Evolution (LTE) system's turbo codes [15]. Additionally, the 156 IRaptor codes will be combined with HARQ in Section IV, 157 where the turbo-coded HARQ of the LTE standard is chosen 158 as the most powerful existing benchmarker. Finally, Section V 159 offers our conclusions.

160

\section{Fountain Codes}

161

Fountain codes are known for its capability of producing 162 an endless supply of encoded symbols for a block of source 163 symbols, each having a length of 1 bit in this paper. They may 164 be visually characterized by a Tanner graph, as shown in Fig. 1, 165 where circles denote the so-called VNs and squares denote CNs. 166

More explicitly, the fountain encoder outputs a sequence 167 of encoded bits $b_{1}, b_{2}, \ldots, b_{N}$ for a block of source bits 168 $a_{1}, a_{2}, \ldots, a_{K}$. Each encoded bit $b_{j}$ is generated by modulo- 2169 adding several randomly chosen source bits $a_{d_{i}}$, where $d_{i} \in \Omega_{j} 170$ and $\Omega_{j}$ is the set formed by all the connections by which the 171 source bits $a_{d_{i}}$ are connected with the encoded bit $b_{j}$, as shown 172 in Fig. 1. Mathematically, each encoded bit is generated by

$$
b_{j}=a_{d_{1}} \oplus a_{d_{2}} \cdots \oplus a_{d_{v}}, \quad\left(d_{1}, d_{2}, \ldots, d_{v} \in \Omega_{j}\right) .
$$

The number of source bits $v$ in the set $\Omega_{j}$ is referred to as 174 the "degree" of that encoded bit, which is a random variable 175 following a specific degree distribution. Furthermore, the po- 176 sitions (represented by $d_{i}$ ) of these source bits involved in 177 generating each encoded bit are evenly distributed in the block 178 region $[1, K]$. 


\section{A. Soft Decoding}

181 LT codes [2] constitute a family of practical fountain codes, 182 which rely on the belief propagation algorithm for simplifying 183 the hard decoding of encoded packets received over BECs. 184 When they act as channel codes in the physical layer, their 185 soft decoding relying on the sum product algorithm [16] based 186 on belief propagation is also feasible for transmissions over 187 noisy fading channels [17]-[19]. Note that only $N_{t}$ successfully 188 received packets are involved in the hard decoding of LT codes 189 received over BECs, whereas the other $\left(N-N_{t}\right)$ encoded 190 packets have been erased owing to their corruption or due to 191 network congestion.

192 However, all $N$ encoded bits will assist the soft decoding of 193 LT codes over noisy fading channels. Explicitly, the receiver 194 is capable of reconstructing the same Tanner graph as the 195 transmitter with the aid of a pseudorandom generator or by 196 using some side information passed along with the transmitted 197 bits. Then, soft information will be iteratively exchanged along 198 the edges between the CNs and VNs of this Tanner graph, as 199 shown in Fig. 1, where $L_{b_{j} \rightarrow a_{i}}^{(m)}$ represents the log likelihood 200 ratios (LLRs) passed from the CNs $b_{j}$ to the VNs $a_{i}$ at the $m$ th 201 iteration, whereas $L_{a_{i} \rightarrow b_{j}}^{(m)}$ represents the LLRs passed from the $202 \mathrm{VNs} a_{i}$ to the $\mathrm{CNs} b_{j}$ at the $m$ th iteration. The following two 203 equations are calculated in turn during the iterative decoding 204 [16], [20]:

$$
\begin{aligned}
& L_{b_{j} \rightarrow a_{i}}^{(m)}=\tilde{b}_{j} \boxplus\left(\sum_{i^{\prime} \in \Omega_{j}, i^{\prime} \neq i} \boxplus\left(L_{a_{i^{\prime}} \rightarrow b_{j}}^{(m-1)}\right)\right) \\
& L_{a_{i} \rightarrow b_{j}}^{(m)}=\sum_{j^{\prime} \in \Psi_{i}, j^{\prime} \neq j} L_{b_{j^{\prime}} \rightarrow a_{i}}^{(m)}
\end{aligned}
$$

205 where $\tilde{b}_{j}$ represents the channel's output LLR corresponding to 206 the encoded bit $b_{j}$ and the set $\Psi_{i}$ is formed of all CNs connected 207 to the single VN $a_{i}$ in a way similar to the set of connections $208 \Omega_{j}$ defined before. Furthermore, $\boxplus$ is referred to as the box209 plus operation, where the box-plus of two LLR values may be 210 specifically expressed as [21]

$$
L_{1} \boxplus L_{2}=\operatorname{sign}\left(L_{1}\right) \cdot \operatorname{sign}\left(L_{2}\right) \cdot \min \left\{\left|L_{1}\right|,\left|L_{2}\right|\right\}+f(\cdot)
$$

211 with $f(\cdot)$ being a correction term, which may be stored 212 in a lookup table (LUT), based on quantized versions of 213 the accurate expression of $\log \left(1+e^{\left(-\left|L_{1}+L_{2}\right|\right)}\right)-\log (1+$ $\left.214 e^{\left(-\left|L_{1}-L_{2}\right|\right)}\right)$. Furthermore, the box-plus operator defined for 215 two LLRs in (4) can be extended to more LLRs by exploiting 216 the associativity of the box-plus operator.

217 Iterative decoding starts from (2), calculating the extrinsic 218 LLRs $L_{b_{j} \rightarrow a_{i}}^{(m)}$ for each edge and for all CNs in Fig. 1. In the first 219 computation of (2), the initial a priori LLRs of $L_{a_{i} \rightarrow b_{j}}^{(0)}$ are set 220 to zeros. The outputs $L_{b_{j} \rightarrow a_{i}}^{(1)}$ will be used as the a priori LLRs 221 in (3). The next evaluation of (3) calculates the corresponding 222 extrinsic LLRs $L_{a_{i} \rightarrow b_{j}}^{(1)}$ for all VNs. This may provide increased 223 a priori LLRs $L_{a_{i} \rightarrow b_{j}}^{(1)}$ fed back to (2) for the second round 224 of calculations. After invoking (2) in each iteration, a hard decision may be made on the a posteriori LLRs of ã, where 225 each element may be calculated as

$$
\tilde{a}_{i}=\sum_{j \in \Psi_{i}} L_{b_{j} \rightarrow a_{i}} .
$$

Then, the iterations continue until a successful recovery de- 227 tected by a CRC or convergence is achieved when no extrinsic 228 information can be obtained.

\section{B. Tanner Graph Analysis}

MacKay explained in [1] that the degree-one CNs play a 231 critical role in the hard decoding of LT codes. He demonstrated 232 that a desired degree distribution should produce many low- 233 degree $\mathrm{CNs}$ to facilitate the commencement of the decoding 234 process and to keep it going while additionally generating a 235 small fraction of high-degree CNs to protect all source packets. 236 However, the actual degree distribution may deviate from the 237 originally designed distribution, unless the block length is suf- 238 ficiently long. For short block lengths, this deviation also leads 239 to a deficient Tanner graph exhibiting an unfavorable structure, 240 as identified by Mackay [1], namely, having "no degree-one 241 CNs," "no emerging degree-one CNs" and "uncovered VNs." 242 At first glance, the LT soft decoding described in Section II-A 243 remains unaffected by the Tanner graph's structure. However, 244 here, we will analyze these structural phenomena of the Tanner 245 graph, which significantly degrade the attainable soft decoding 246 performance of fountain codes for transmission over noisy 247 fading channels, albeit they have neither been mentioned in 248 [6]-[11] nor been explicitly exploited by MacKay.

249

1) No Degree-One CNs: By examining (4), we can infer that 250 its result can only be a valid nonzero value, when both $L 1$ and 251 $L 2$ are nonzero. Therefore, initially only the channel LLRs $\tilde{b}_{j} 252$ connected to a degree-one $\mathrm{CN}$ may be passed to the edges of the 253 Tanner graph by observing (2), since all initial LLRs $L_{a_{i} \rightarrow b_{j}}^{(0)} 254$ have a value of zero. Then, the iterative LT soft decoding 255 process has to be triggered by at least one degree-one CN. 256

2) No Emerging "Degree-One" CNs: The success of seam- 257 lessly continued iterations depends on encountering at least one 258 new "degree-one" CN emerging at each iteration. This "degree- 259 one" CN is not a real degree-one CN connected to a single 260 VN as stated in [1]. Instead, there are some CNs in the Tanner 261 graph that have a degree higher than one in the presence of zero 262 a priori LLRs, i.e., no a priori information. However, when 263 all a priori LLRs along all other edges that are involved for 264 calculating the extrinsic LLR of one of the edges of this CN 265 become nonzero, these corresponding input edges of this $\mathrm{CN} 266$ may be deemed to be removed. Hypothetically speaking, we 267 may be left with a single edge awaiting the calculation of its 268 extrinsic LLR at this particular CN. Hence, we might refer to 269 these in quotation mark, as "hypothetical degree-one" CNs in 270 the soft decoding.

For example, assume that the encoded bit $b_{1}$ has an original 272 degree of two, connecting the source bit $a_{1}$ and $a_{2}$. On eval- 273 uating $L_{b_{1} \rightarrow a_{1}}^{(2)}=\tilde{b}_{1} \boxplus L_{a_{2} \rightarrow b_{1}}^{(1)}$ in the second iteration, $L_{a_{2} \rightarrow b_{1}}^{(1)} 274$ will act as the a priori LLR, provided that it becomes a 275 nonzero value after evaluating (3) in the first iteration. Since all 276 


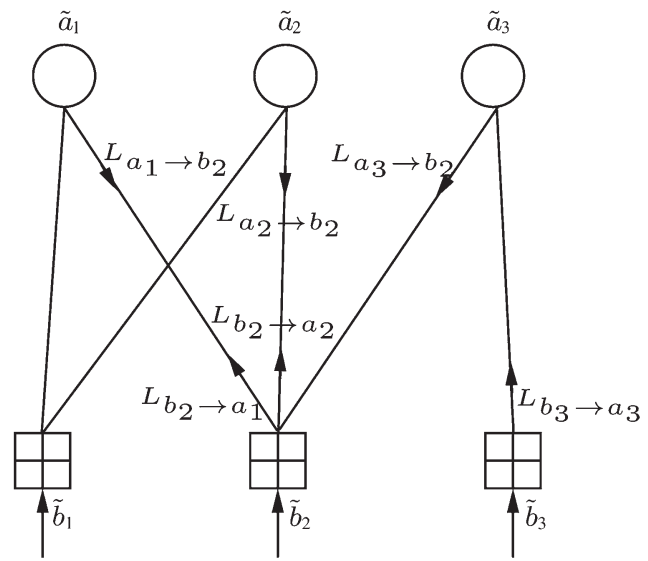

Fig. 2. Tanner graph where no new "degree-none" $\mathrm{CN}$ will emerge at the second iteration.

277 operands are nonzero here, an increased nonzero $L_{b_{1} \rightarrow a_{1}}^{(2)}$ may 278 be obtained. In this way, the channel LLR $\tilde{b}_{1}$ may be invoked 279 within the Tanner graph for assisting the rest of the iterative 280 decoding process.

281 Nonetheless, there exists a specific Tanner graph structure, 282 where no new "hypothetical degree-one" CNs appear at some 283 stage; hence, the remainder of the CNs are left without a "hy284 pothetical degree-one" node, and as a result, the corresponding 285 channel LLRs become "helpless." Fig. 2 shows an example of 286 this structure, where the decoding performance fails to improve 287 after the first iteration. No new nonzero LLRs may be obtained 288 during the second iteration or in fact not even during the 289 ensuing later iterations. In this example, The channel LLRs $\tilde{b}_{1}$ 290 and $\tilde{b}_{2}$ are never entered into the graph; hence, they become 291 unexploited, i.e., useless.

292 3) Uncovered VNs: As aforementioned, a small fraction of 293 high-degree CNs is likely to avoid the absence of some source 294 bits among the encoded bits, albeit this increases the fountain 295 codes' decoding complexity. Hence, Raptor codes [3] invoke 296 LDPC codes as their outer code for decoding the uncovered 297 source bits, so that the inner LT code may adopt a component 298 degree distribution imposing reduced complexity.

299 Despite the lower complexity of the inner LT codes, Raptor 300 codes' recent studies [3], [8], [9] ignore the LDPC decoding 301 complexity, although in this light their complexity contribution 302 is substantial. Moreover, the degree distribution of the inner LT 303 codes in [3] also contains several terms having a high degree, 304 e.g., the degree terms of 64 and 65 . Therefore, the overall 305 complexity of Raptor codes may become unattractive.

\section{IMPROVED FOUNTAIN CODES}

307 Systematic fountain codes [14] automatically and naturally 308 solve the problems of having "no degree-one CNs" and "un309 covered VNs" in the Tanner graph by transmitting all the 310 original information bits before the fountain encoded parity 311 bits. However, the problem of having "no emerging degree-one 312 CNs" - as mentioned in Section II-B — still exists in systematic 313 fountain codes.
TABLE I

RECORDED LISTS FOR THE TANNER GRAPH OF Fig. 2

\begin{tabular}{|c||c|}
\hline CN index & Lists \\
\hline 1 & $\{1,2\}$ \\
\hline 2 & $\{1,2,3\}$ \\
\hline 3 & $\{3\}$ \\
\hline \hline VN index & Lists \\
\hline 1 & $\{1,2\}$ \\
\hline 2 & $\{1,2\}$ \\
\hline 3 & $\{2,3\}$ \\
\hline
\end{tabular}

For nonsystematic fountain codes, regardless of how good 314 the channel conditions are, the attainable performance may be 315 degraded by the above three potential features of the Tanner 316 graph. Based on the fact that the encoder and decoder rely 317 on the same Tanner graph, these structural phenomena may 318 be eliminated by the encoder since it is potentially capable of 319 adjusting the Tanner graph structure after generating a specific 320 number of encoded bits.

\section{A. Solutions for Improving the Tanner Graph}

To realize this Tanner graph construction, we allocate a list 323 for each VN and each $\mathrm{CN}$ to record the indexes of its connected 324 counterparts. As for the lists of CNs, they are simply formed 325 once an encoded bit is created, whereas the lists of VNs are 326 dynamically filled during the generation of each encoded bit. 327 For example, when considering the Tanner graph of Fig. 2, the 328 list of CN 1 is $\{1,2\}$, storing the indexes of its connected VNs 329 when CN 1 is generated. Simultaneously, this CN's list com- 330 ponent at index 1 is in turn appended to the lists of those VNs 331 that it is connected to, namely to VN 1 and VN 2 in our specific 332 example. Finally, once three encoded bits have been generated, 333 all recorded lists are as shown in Table I.

With the aid of these lists, we are now ready to solve the 335 potential problems caused by the Tanner graph's structure. Af- 336 ter a number of encoded bits have been generated, the encoder 337 may readily spot the occurrence of "no degree-one CNs" and 338 "uncovered VNs" by inspecting the recorded lists of CNs and 339 VNs. To eliminate the occurrence of "no degree-one CNs," 340 the encoder will generate a new degree-one $\mathrm{CN}$ in the usual 341 way. For eliminating "uncovered VNs," the encoder produces 342 the corresponding number of CNs with a random degree plus 343 one, where the random degree indicates the VNs randomly 344 selected with an identical probability, whereas the additional 345 one corresponds to the one among the uncovered VNs. $\quad 346$

The "no emerging degree-one CNs" situation may also be 347 found by canceling the nodes and their connected edges from 348 the Tanner graph one by one. More explicitly, this cancelation 349 commences from any of the current degree-one CNs. Using the 350 illustrative lists in Table I as an example, the degree-one CN 3351 will be first removed, which is arranged by deleting the contents 352 of its list $\{3\}$, making it an empty list. Nonetheless, the list $\{3\} 353$ of $\mathrm{CN} 3$ before cancelation implies that the resultant belief may 354 be propagated along the edge to $\mathrm{VN} 3$ during the decoding. 355 Then, based on the list $\{2,3\}$ of VN 3 , the corresponding belief 356 is conveyed back to $\mathrm{CN} 2$. We refer to this as a completed belief 357 propagation round, commencing from a degree-one $\mathrm{CN}$ and 358 flowing back to several other CNs, whose degree will be less 359 


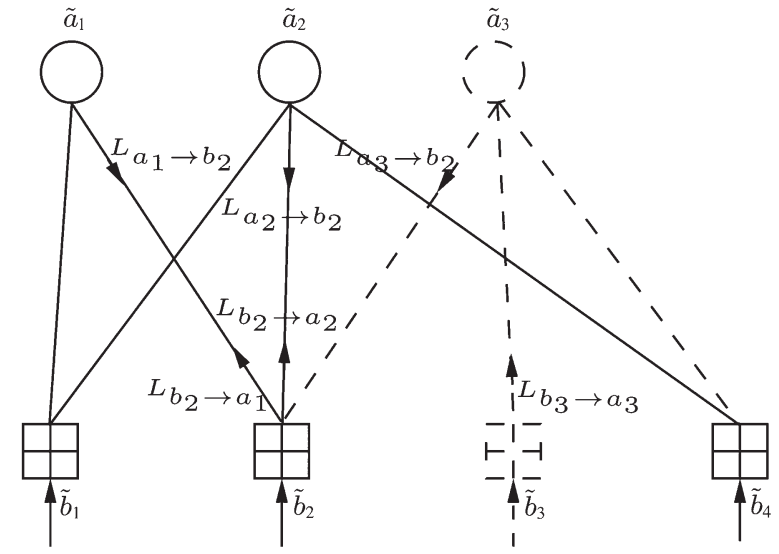

Fig. 3. Enhanced Tanner graph of Fig. 2 generated by adding the extra CN 4, which has a degree of two connecting the just-canceled VN 3 and the VN 2. The VN 2 is connected to $\mathrm{CN} 1$, which has the lowest degree of two among the remainder of the CNs. Here, the parts drawn in the dashed line indicate that they are canceled from the Tanner graph during the process of checking and resolving the structural phenomenon of "no emerging degree-one CNs."

360 by one. More explicitly, after this completed belief propagation 361 round, the list of VN 3 is also made an empty list, and all edges 362 connected to VN 3 have to be removed from its connected CNs. 363 In this case, its connected CN 2 will remove the index 3 from its 364 list, changing the list from $\{1,2,3\}$ to $\{1,2\}$. In other words, 365 the degree of $\mathrm{CN} 2$ has been decreased from 3 to 2 since the 366 number of indexes in a CN's list indicates that particular CN's 367 degree.

368 Then, we have to check, whether a new degree-one CN 369 emerges. If a new degree-one $\mathrm{CN}$ appears, the same list-nulling 370 actions will be repeated. Otherwise, a new $\mathrm{CN}$ needs to be 371 generated, attempting to overcome the current structural phe372 nomenon. Note that the degree of this new $\mathrm{CN}$ is not randomly 373 chosen as that of a conventional fountain encoded bit. Instead, 374 it is set to be a degree of two to supply an emerging degree-one $375 \mathrm{CN}$ in succession. In detail, the new $\mathrm{CN}$ will have two edges, 376 one of which connects it to the just canceled VN, whereas the 377 other connects it to any one of the VNs, which are connected 378 to the lowest degree $\mathrm{CN}$ in the remainder of the Tanner graph. 379 For the example of Fig. 2, the new degree-2 CN is connected to 380 the just-canceled VN 3, as well as to VN 2, which is connected 381 to the current lowest degree $\mathrm{CN}$ 1, as shown in Fig. 3. It may 382 be seen that the belief propagation may continue to flow, after 383 producing this new $\mathrm{CN} \mathrm{4,} \mathrm{which} \mathrm{is} \mathrm{supposed} \mathrm{to} \mathrm{have} \mathrm{a} \mathrm{list} \mathrm{of}$ $384\{2,3\}$. Yet, its shortened list $\{2\}$ generated after the removal 385 of the index of 3 will be added to the rest of the Tanner graph 386 for triggering the next cancelations since the VN 3 has been 387 canceled at this moment.

388 In conclusion, the general process of eliminating the phe389 nomenon of encountering 'no emerging degree-one CNs' en390 sues as follows.

391

- Step 1: Attempt to find a degree-one CN. If none exists, go to Step 3.

- Step 2: Cancel the just identified degree-one $\mathrm{CN}$, its connected VN, and all edges of that VN. Then, go to Step 4.
- Step 3: Generate a new degree-two CN, connecting to 397 the just-canceled VN and any one of the VNs connecting 398 to the specific $\mathrm{CN}$, which has the lowest degree in the 399 remainder of the Tanner graph.

- Step 4: Loop to Step 1 until the Tanner graph becomes 401 empty, i.e., it is eliminated.

402

When the proposed strategies are applied to the fountain 403 codes for solving the structural phenomena of Tanner graphs, 404 the high-degree terms of the degree distribution may be left out 405 for the sake of reducing the average degree since there is no need 406 to worry about uncovered VNs. As discussed in Section II-B3, 407 having high-degree terms in the degree distribution reduces 408 the probability of having uncovered VNs for nonsystematic 409 fountain codes. Therefore, in our simulations discussed in 410 Section III-B, we adopt the following degree distribution for 411 our improved fountain codes:

$$
\begin{array}{r}
\delta(x)=0.091717+0.493570 x+0.166220 x^{2}+0.072646 x^{3} \\
+0.082558 x^{4}+0.056058 x^{7}+0.037229 x^{8}
\end{array}
$$

which was simply formed by adding the weighting coefficients 413 representing the high-degree terms in the degree distribution of 414 Raptor codes in [3] to the weighting coefficient of the degree- 415 one term.

Clearly, we have improved the performance of fountain 417 codes by transmitting a few specifically encoded bits. However, 418 another practical issue has risen: When should the encoder 419 generate these encoded bits during the continuous fountain 420 encoding process? In Section III-B, we chracterized our IRaptor 421 codes based on a fixed coding rate, hence naturally followed 422 by these specifically encoded bits. Furthermore, we proposed 423 a novel IRaptor-coded HARQ scheme detailed in Section IV, 424 where a LUT prestores the most appropriate coding rates for 425 IRaptor codes corresponding to the different channel SNRs 426 of each (re)transmission. Then, these specifically encoded bits 427 can be generated right after the required coding rate has been 428 reached during each (re)transmission.

\section{B. Verifying IRaptor Codes}

To characterize the performance of our improved fountain 431 codes, both the PLR achieved and the complexity imposed 432 will be compared with those of conventional fountain codes. 433 We investigated 1) nonsystematic LT codes (LT); 2) systematic 434 Raptor codes (sys Raptor); 3) nonsystematic Raptor codes 435 (Raptor); 4) improved nonsystematic LT codes (ILT); and 436 5) our improved nonsystematic Raptor codes (IRaptor), ${ }^{1}$ by col- 437 lecting the statistical results after a relevant number of blocks 438 were transmitted, using binary phase-shift keying (BPSK) 439 modulation for transmissions over an additive white Gaussian 440 noise (AWGN) channel. Furthermore, the LTE turbo codes are 441 also used as the most powerful existing benchmarker in our 442 simulations for the sake of comparing its PLR performance 443

\footnotetext{
${ }^{1}$ We did not involve systematic LT codes in our simulations since systematic Raptor codes have included an inner systematic LT code and shown higher performance than sole systematic LT codes.
} 
444 with our IRaptor codes. The short block lengths of 104 and 4451008 bits, and the longer length of 4992 bits are considered 446 in our simulations.

447 The conventional LT codes adopted the classical degree 448 distribution in [3], whereas our improved LT codes relied on (6). 449 For all conventional and IRaptor codes, the coding rate of the 450 outer LDPC codes was set to a high value of 0.9 . We employed 451 regular systematic LDPC codes using the parameter set of $452(M, 3,30)$, where $M$ is the number of intermediate bits after 453 the outer LDPC encoding, and 3 is the VN's degree, whereas 45430 is the CN's degree in the regular-LDPC Tanner graph. More 455 specifically, the $M$ values for the block lengths of 104 and 4561008 bits are 116 and 1120 , according to the LDPC coding rate 457 of 0.9 . The LDPC codes also employ the sum-product algorithm 458 based on the Tanner graph for decoding [16].

459 We investigated the performance of these five fountain codes 460 having a fixed coding rate and in conjunction with gradually 461 decreasing coding rates. For the sake of a fair comparison, the 462 overall coding rate of the five fountain codes needs to be kept 463 the same. Hence, the coding rate of the sole LT codes was set 464 to 0.45 , whereas the coding rate of the inner LT codes of the 465 Raptor codes was set to 0.5 , which is multiplied with the outer 466 0.9-rate LDPC code rate to have the same overall coding rate 467 of 0.45 . Following the conventional fountain encoding process, 468 our improved fountain codes transmit a series of encoded bits 469 until they reach the aforementioned preset coding rate. Then, 470 the encoder reconstructs the Tanner graph using the recorded 471 lists and generates several specifically encoded bits to circum472 vent the aforementioned structural phenomena, as discussed 473 in Section III-A. Compared with the conventional fountain 474 encoded bits, these extra encoded bits are special since their 475 degree and the VNs involved in the XOR operations are des476 ignated, instead of being randomly selected. The receiver uses 477 a pseudorandom generator or side information along with these 478 specifically encoded bits for maintaining synchronization. Nev479 ertheless, our simulation results demonstrated that the influence 480 of these extra encoded bits on the coding rate can be ignored; 481 hence, it remains close to 0.45 . Specifically, the coding rate of 482 the improved fountain codes is 0.4498 in our simulations.

483 The LTE turbo codes adopt two parallel concatenated con484 volutional codes (CCs) having memory-3 polynomials of $485(15,13)_{o}$ in octal representation. The two CC encoders output 486 the parity bit sequences $b_{1}$ and $b_{2}$, respectively, for the sys487 tematic bit sequence a and its interleaved copy. After a series 488 of interleaving operations specified by the LTE standard [15], 489 the interleaved sequence $\mathbf{a}$ followed by the interleaved and 490 combined sequences $\mathbf{b}_{1}$ and $\mathbf{b}_{2}$ enter into a circular buffer. The 491 encoded bits are one by one taken from the start position of 492 the circular buffer, until the number satisfies the coding rate 493 of 0.45 . The turbo decoder performs the iterative decoding 494 after deinterleaving and depuncturing the received encoded bits, 495 reinstating them into the appropriate bit positions.

496 For all LT codes and the LTE turbo codes [15], except for 497 the cyclic redundancy check (CRC), the early stopping strategy 498 proposed in [22] was employed for terminating the iterative soft 499 decoding. More specifically, instead of using a fixed number of 500 iterations, the decoder will halt the decoding operations once 501 the incremental contribution of the mutual information (MI) to the extrinsic LLRs of VNs becomes less than 0.0001, as 502 represented by $\operatorname{MI}\left(L_{a_{i} \rightarrow b_{j}}^{(m)}\right)-\operatorname{MI}\left(L_{a_{i} \rightarrow b_{j}}^{(m-1)}\right)<0.0001$. This is 503 because using more iterations fails to improve the performance 504 but dissipates more battery power. The function $\mathrm{MI}(\cdot)$ is fur- 505 ther detailed in [23, Sec. 2.3], which estimates the MI of the 506 extrinsic LLR sequence $L_{a_{i} \rightarrow b_{j}}$. There are two Tanner graphs 507 in Raptor codes, where one of them is characterizing the inner 508 LT decoding, whereas the other visualizes the outer LDPC 509 decoding operations. More explicitly, following $I_{\text {inner }}$ a number 510 of iterations within the inner LT decoding loops, the output 511 a posteriori LLRs are subjected to a number of sum-product 512 algorithm iterations performed by the outer LDPC decoder. The 513 given early stopping strategy is also employed for terminating 514 the inner iterative LT decoding, while we invoke $I_{\text {outer }}=5515$ iterations for the outer LDPC decoding, to control the overall 516 complexity. Nevertheless, the iterative LDPC decoding may not 517 have to be invoked at all if the systematic bits representing the 518 original information bits have already satisfied the CRC-based 519 error checking after the inner iterative LT decoding.

Each node in a Tanner graph is related to addition and box- 521 plus calculations. The complexity of fountain codes may be 522 quantified in terms of the total number of addition and box-plus 523 operations per source bit, which are denoted by $N_{+}$and $N_{\boxplus}, 524$ respectively. Note that the total number of operations indicates 525 the number of additions and box-pluses performed during all 526 iterations, not only for the LT codes but for the LDPC codes as 527 well, when considering the complexity of Raptor codes. When 528 relying on the LUT implementation of $f(\cdot)$ in (4), the com- 529 plexity of box-plus is approximately equal to $\left[5+2 \cdot \log _{2}(T)\right] 530$ additions, ${ }^{2}$ where $T$ is the number of entries in the LUT. 531 Referring to [24], when the number of entries becomes $T \geq 4,532$ the performance of correction-factor-aided min-sum decoding 533 approaches that of the optimum sum-product algorithm, while 534 maintaining low complexity. Therefore, the expression of $(9 \times 535$ $N_{\boxplus}+N_{+}$) may accurately reflect the complexity of fountain 536 codes. This makes it possible for us to compare the complexity 537 of LT codes and Raptor codes.

538

Given these simulation parameters, Fig. 4(a)-(c) shows the 539 PLR performance for a block length of 104, 1008, and 4992 bits, 540 respectively. Furthermore, Fig. 5(a)-(c) portray the correspond- 541 ing complexity for three block lengths, where the complexity of 542 all schemes was divided by 1000 for convenient plotting. $\quad 543$

For the block length of 104 bits, it may be observed in 544 Fig. 4(a) that our IRaptor codes achieve the lowest PLR among 545 all fountain codes, while having significantly lower complexity 546 compared with the conventional fountain codes. More specif- 547 ically, the PLR curve of the IRaptor codes decays rapidly, 548 approaching a vanishingly low value upon increasing the SNR. 549 At a PLR of $10^{-3}$, our scheme requires $1.5 \mathrm{~dB}$ lower SNR than 550 that of the systematic Raptor codes, which have the best per- 551 formance among the conventional fountain codes. Furthermore, 552 observe in Fig. 4(a) that our improved fountain codes substan- 553 tially outperform the nonsystematic fountain codes since the 554

\footnotetext{
${ }^{2}$ The number of clock cycles performing a single box-plus operation in (4) includes five cycles for comparison operations for obtaining the minimum value between $L_{1}$ and $L_{2}$ and includes twice searching of the LUT, each of which consumes $\log _{2}(T)$ cycles.
} 


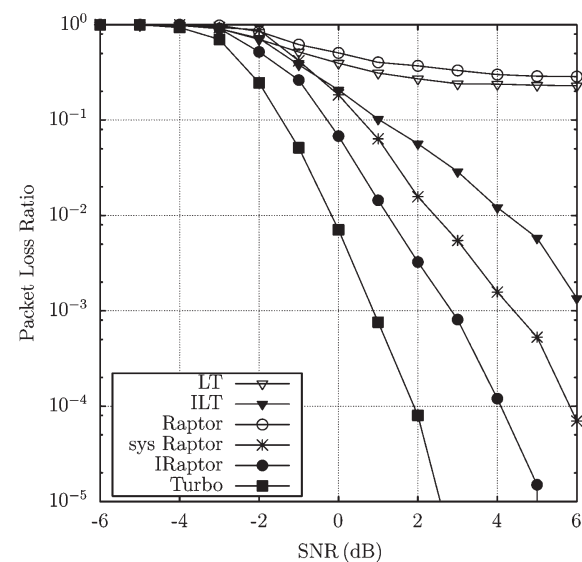

(a)

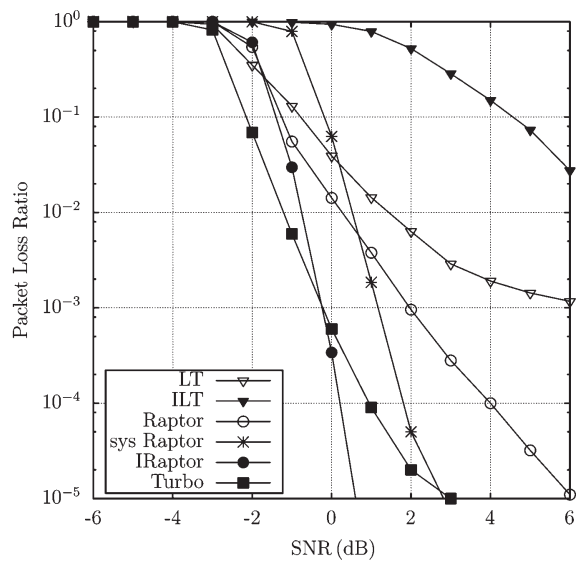

(b)

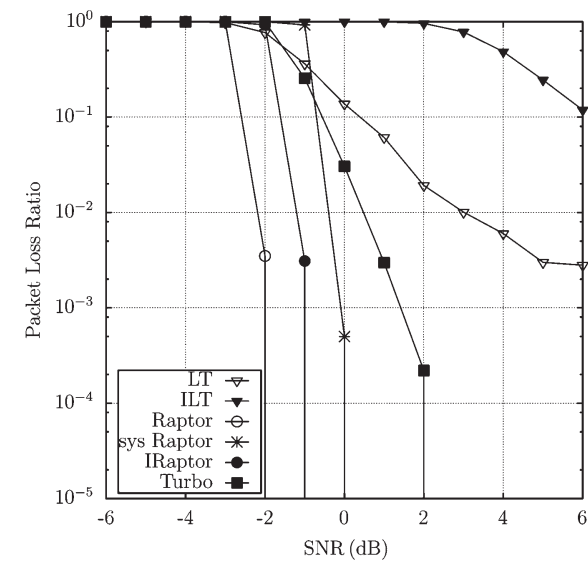

(c)

Fig. 4. PLR versus the AWGN channel SNR for the block lengths of (a) 104, (b) 1008, and (c) 4992 bits.

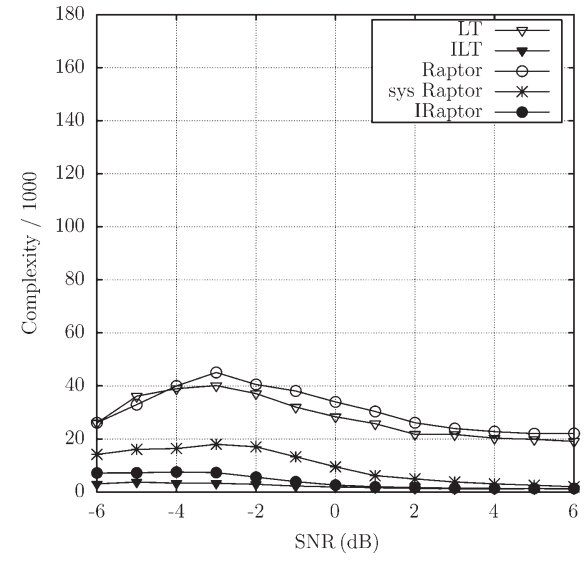

(a)

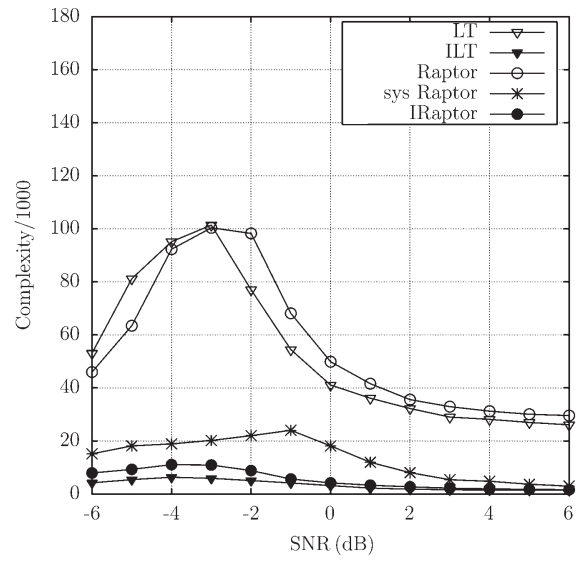

(b)

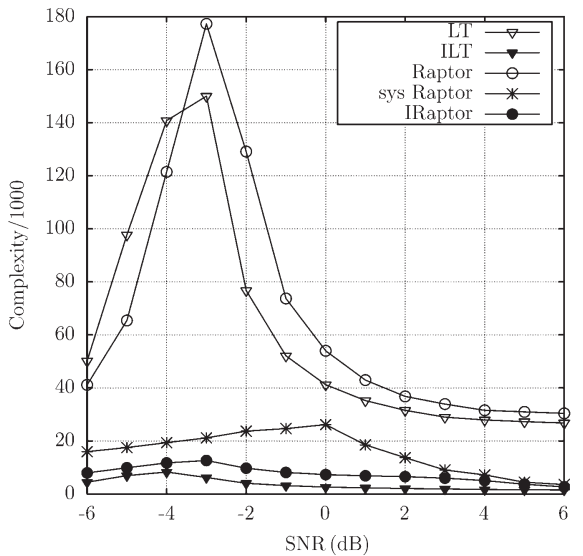

(c)

Fig. 5. Complexity versus the AWGN channel SNR for the block lengths of (a) 104, (b) 1008, and (c) 4992 bits.

555 latter codes have a PLR in excess of 0.2 even at high SNRs. 556 As for the complexity characterized in Fig. 5(a), the improved 557 LT codes impose the lowest complexity among the five fountain 558 codes. The complexity of the IRaptor codes is slightly higher 559 than that of the improved LT codes, owing to the additional 560 LDPC decoding complexity. However, the IRaptor codes still 561 exhibit dramatically reduced complexity, which is less than 562 half of the systematic fountain codes as a benefit of the low563 complexity degree distribution of (6).

564 Although the improved LT code fails to excel for the 1008-bit 565 block length characterized in Fig. 4(b) in terms of its PLR, it 566 imposes the lowest complexity, as shown in Fig. 5(b). This is 567 because the straightforward degree distribution of (6) may not 568 be the optimal one. A low fraction of errors persists in each 569 block after the decoding of the improved LT codes. Never570 theless, these residual errors are likely to be corrected by the 571 outer 0.9-rate LDPC code. Therefore, the IRaptor codes exhibit 572 the best PLR performance among the five fountain codes, as 573 evidenced by Fig. 4(b). The IRaptor codes attain a PLR of $10^{-5}$ 574 at the SNR of $0 \mathrm{~dB}$, which is $2 \mathrm{~dB}$ lower than that required by 575 the conventional systematic Raptor codes and $6 \mathrm{~dB}$ lower than 576 the SNR of the nonsystematic Raptor codes. Simultaneously, 577 as shown in Fig. 5(b), the complexity of IRaptor codes is only about half of the conventional systematic Raptor codes. 578 Compared with the conventional nonsystematic fountain codes, 579 the complexity imposed may be reduced by as much as an order 580 of magnitude.

Although our proposed scheme initially aimed for short 582 lengths, our IRaptor codes actually exhibit a desirable per- 583 formance for the longer 4992-bit codewords. As shown in 584 Fig. 4(c), they have a PLR versus SNR curve that is $1 \mathrm{~dB}$ better 585 than that of the systematic Raptor codes, even though it is $1 \mathrm{~dB} 586$ worse than that of the conventional Raptor codes. However, 587 our IRaptor codes achieve the lowest complexity among these 588 three codes, as shown in Fig. 5(c). More explicitly, Fig. 5(c) 589 demonstrates that the complexity of the IRaptor codes may be 590 ten times lower than that of the conventional Raptor codes. 591 Compared with the systematic Raptor codes, the complexity 592 may also be reduced by as much as a factor of 3.5 by our 593 IRaptor codes.

The PLR trend of the LTE turbo codes [15] is also presented 595 in Fig. 4. It can be observed that, in Fig. 4(a), that the LTE turbo 596 code associated with the 104-bit block length outperforms our 597 IRaptor code by about $2 \mathrm{~dB}$. However, for the block length of 598 1008 bits, it is observed in Fig. 4(b) that the PLR curve of our 599 IRaptor code decays more rapidly than that of the LTE turbo code 600 


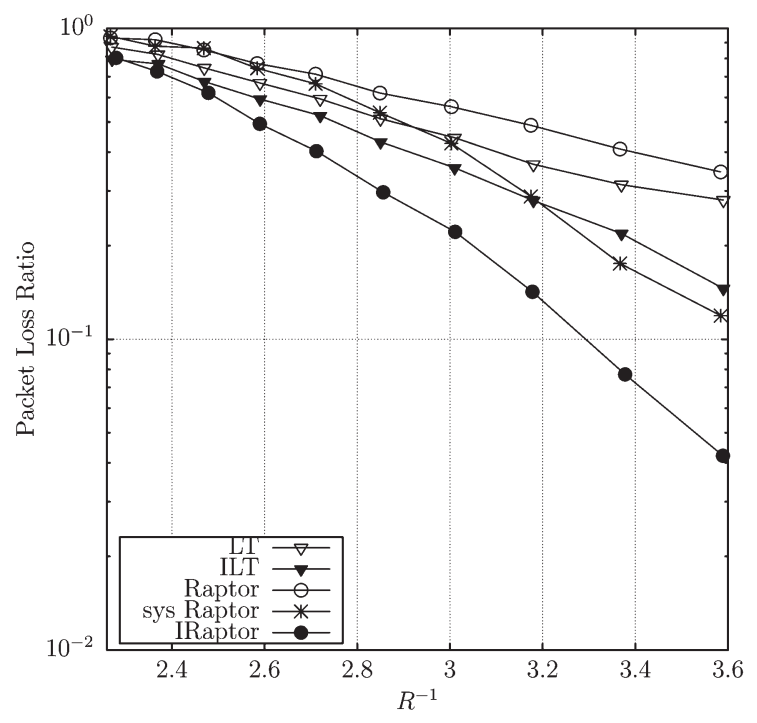

(a)

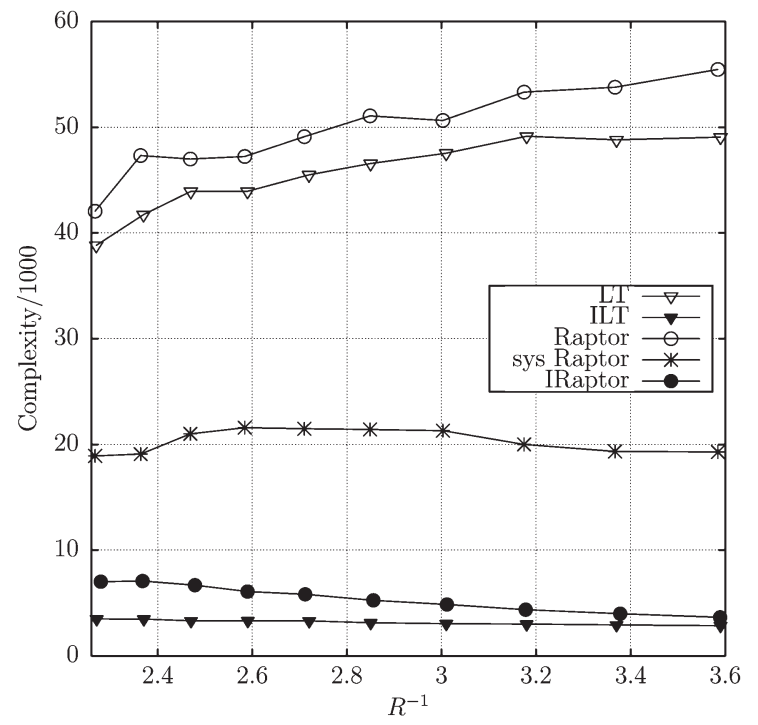

(b)

Fig. 6. (a) PLR and (b) complexity versus the reciprocal of the coding rate for the 104-bit block length at the SNR of $-3 \mathrm{~dB}$ for transmission over AWGN channels.

601 and exhibits a significantly lower error floor. More specifically, 602 the PLR of our IRaptor codes becomes lower than that of the $603 \mathrm{LTE}$ turbo codes for SNRs in excess of $0 \mathrm{~dB}$, as shown in 604 Fig. 4(b). Furthermore, the PLR versus SNR advantage of our 605 IRaptor codes increases upon increasing the codeword length, 606 which becomes $3 \mathrm{~dB}$ better than that of the LTE turbo codes, as 607 shown in Fig. 4(c) for the 4992-bit codewords.

608 The PLR performance of Fig. 4(b) and (c) confirms that our 609 IRaptor codes may be capable of outperforming the LTE turbo 610 codes when the block length is in excess of 1008 bits, whereas 611 they are outperformed by the LTE scheme in Fig. 4(a) for the 612 short block length of 104 bits. We will further explore the 613 theoretical reason behind this phenomenon in our future work. 614 Note that it is always a challenge to compare two different 615 types of channel codes since a diverse range of contradictory 616 factors should be taken into account, as shown in [25, Fig. 1.3], 617 including the BER/PLR, the complexity, the coding delay, the 618 throughput, etc., and the specific channel characteristics for 619 which the code was designed. In Section IV, a fairer com620 parison between the LTE turbo codes and our IRaptor codes 621 will be offered in a practical environment, when both of them 622 are combined with an adaptive HARQ scheme, where various 623 coding rates are required for each transmission encountered 624 with different channel conditions in a quasi-static Rayleigh 625 fading channel mode.

626 Additionally, we explored the candidates' behavior at dif627 ferent coding rates. Fig. 6(a) illustrates the PLR performance 628 versus the reciprocal of the coding rate for the 104-bit block 629 length at the SNR of $-3 \mathrm{~dB}$, demonstrating that the IRaptor 630 codes have a significantly lower PLR than the other foun631 tain codes. Specifically, as shown in Fig. 6(a), when the IRaptor 632 codes achieve a PLR of 0.1 , the overall coding rate has to be 633 about $1 / 3.3=0.303$. By contrast, the systematic Raptor codes 634 exhibit the best performance among the conventional fountain 635 codes, requiring a coding rate of about $1 / 3.65=0.274$ at the 636 PLR of 0.1. Meanwhile, Fig. 6(b) reveals that the complexity of the IRaptor codes is a factor of three lower than that of the 637 systematic Raptor codes and an order of magnitude lower than 638 that of the nonsystematic fountain codes.

639

In conclusion, the simulation results here have demonstrated 640 that the IRaptor codes achieve the lowest PLR, despite having 641 lower complexity than that of the conventional fountain codes. 642 This is achieved at the cost of a modestly increased memory 643 requirement during the encoding process at the transmitter. 644 More specifically, this memory is used for storing the lists of all 645 VNs and CNs, whereas a few additional operations are involved 646 in going through these lists for generating several specifically 647 encoded bits, as discussed in Section III-A.

\section{AdAPTIVE IRAPTOR-CODED Hybid AUtomatiC REPEAT REQUEST}

Traditional HARQ has a fixed coding rate for each of its 651 (re)transmissions, each of which may encounter different chan- 652 nel conditions when a quasi-static Rayleigh fading channel 653 model is considered. Therefore, a fixed-rate HARQ scheme 654 may not be capable of making an efficient use of the spe- 655 cific channel conditions that occur within all (re)transmissions. 656 Moreover, the retry limit of the fixed-rate HARQ constrains the 657 overall coding rate, which may result in unsuccessful decoding, 658 when the channel conditions of all (re)transmissions are hostile. 659 In this case, the packet will be deemed lost despite all the 660 (re)transmissions. Hence, both the PLR and the throughput 661 suffer.

The straightforward solution is to use an adaptive HARQ 663 scheme, which may invoke different coding rates for each 664 (re)transmission, bearing in mind the specific channel condi- 665 tions encountered. This requires that the transmitter should 666 know the channel quality before its transmission and that the 667 channel code is capable of supporting arbitrary coding rates. 668 In the LTE system [15], CQI messages are used for indicating 669 the channel states for appropriately configuring its adaptive 670 
TABLE II

Coding Rates of IRAPtor Codes, Aiming For A PLR of 0.1 When the PaCket Length IS 104-bit

\begin{tabular}{c||c||c||c||c||c||c||c||c||c||c|}
\hline SNR(dB) & -12.0 & -11.0 & -10.0 & -9.0 & -8.0 & -7.0 & -6.0 & -5.0 & -4.0 \\
\hline $\mathrm{R}$ & 0.05 & 0.07 & 0.08 & 0.10 & 0.13 & 0.16 & 0.2 & 0.24 & 0.29 \\
\hline \hline SNR(dB) & -3.0 & -2.0 & -1.0 & 0.0 & 1.0 & 2.0 & 3.0 & 4.0 & 5.0 & 6.0 \\
\hline $\mathrm{R}$ & 0.34 & 0.41 & 0.47 & 0.54 & 0.61 & 0.68 & 0.75 & 0.82 & 0.89 & 1 \\
\hline
\end{tabular}

671 modulation scheme before transmission. They may be ben672 eficially utilized by our IRaptor-coded HARQ scheme pro673 posed here by exploiting its rateless property. The minimum 674 required number of encoded symbols was theoretically studied 675 for a Raptor-coded HARQ scheme in [26]. By contrast, in our 676 IRaptor HARQ, a LUT will be designed in the following for 677 prestoring all the required coding rates.

\section{A. LUT Design}

679 Again, we carefully design a LUT for the IRaptor-coded 680 HARQ scheme, which prestores the coding rates of IRaptor 681 codes required for different SNRs. Using these coding rates, 682 a PLR of about 0.1 may be achieved for transmissions over 683 an AWGN channel. This LUT may be generated by an offline 684 training process, which relies on transmitting a sufficiently high 685 number of IRaptor encoded packets at various coding rates, as 686 described in Section III-B. Table II shows an example of the 687 LUT designed for the SNR range spanning from -12 to $6 \mathrm{~dB}$ 688 for the packet length of 104 bits.

689 According to the LUT shown in Table II, a low coding rate 690 is required for guaranteeing a high probability of successful re691 ception, if a specific transmission attempt occurs in a deep fade. 692 For example, a coding rate as low as 0.05 is required for the $693 \mathrm{SNR}$ of $-12 \mathrm{~dB}$. This may however significantly degrade the 694 effective throughput. If a HARQ scheme avoids transmissions 695 in deep fades, and instead it opts for transmitting during better 696 channel conditions within the allowed retransmission limit, it 697 will achieve both a high effective throughput and a desirable 698 PLR performance.

\section{B. HARQ Flow}

700 Based on the given analysis, our proposed IRaptor-coded 701 HARQ deactivates potentially futile transmissions when the 702 channel experiences a deep fade, or continues transmitting, 703 until the number of IRaptor encoded bits becomes capable 704 of guaranteeing a low PLR. The channel condition may be 705 identified by a short CQI message, which characterizes the SNR 706 estimated by the receiver.

707 A short CQI message carrying the estimated SNR will be fed 708 back from the receiver to the transmitter before its next trans709 mission. If the estimated SNR is higher than a threshold SNR $710 \sigma_{\mathrm{th}}$, the transmitter will search through the LUT in order to find 711 the most appropriate coding rate. Then, it continues transmit712 ting the IRaptor encoded bits, until that specific coding rate has 713 been reached. After that, it will reconstruct the Tanner graph 714 and transmits several specifically encoded bits for overcoming 715 its potential structural phenomena, as described in Section III-A. 716 Otherwise, if the estimated SNR is lower than $\sigma_{\mathrm{th}}$, the trans717 mitter waits to time out by disabling this transmission. The next retransmission may then be triggered in this situation. 718 The retransmission may also be triggered without receiving an 719 acknowledgement (ACK) message from the receiver until the 720 retry limit is reached.

The receiver is responsible for detecting the channel quality 722 and for sending the CQI message back. It will then execute 723 iterative decoding based on all the received encoded bits. If the 724 CRC is satisfied, the receiver sends an ACK message back to 725 the transmitter; otherwise, it waits for the next retransmission 726 or discards the packet if the maximum retransmission limit is 727 exhausted.

The threshold SNR $\sigma_{\text {th }}$ is used for indicating a deep fade. Its 729 value strikes a tradeoff between the attainable throughput and 730 the PLR encountered, where the throughput is defined as a ratio 731 of the number of successfully received information bits over the 732 total number of transmitted bits from the upper layer's perspec- 733 tive. If the threshold SNR $\sigma_{\text {th }}$ is set to a low value, the packets 734 may be delivered even in a deep fade, but this results in a low 735 coding rate. Hence, it guarantees a low PLR, but as a price, the 736 attainable throughput suffers. By contrast, for a high $\sigma_{\text {th }}$ value, 737 transmission opportunities may be missed even for transmis- 738 sion over a relatively benevolent channel when the estimated 739 SNR is below $\sigma_{\mathrm{th}}$. For example, let us consider the threshold 740 SNRs of $\sigma_{\mathrm{th}}=-12 \mathrm{~dB}$ and $\sigma_{\mathrm{th}}=-8 \mathrm{~dB}$, as well as the esti- 741 mated SNR of $-10 \mathrm{~dB}$. Then, transmissions are avoided for 742 $\sigma_{\mathrm{th}}=-8 \mathrm{~dB}$, since we have $-10<-8$. By contrast, for $\sigma_{\mathrm{th}}=743$ $-12 \mathrm{~dB}$, we have $-10>-12$, hence, the packet's transmission 744 may become successful when using the low coding rate of 0.08745 corresponding to $-10 \mathrm{~dB}$, as shown in Table II.

\section{Simulation Results}

We developed simulations for investigating both the through- 748 put and PLR trends of our proposed IRaptor-coded HARQ 749 scheme for the threshold SNR values in the set of $\{-12,-10,750$ and $-8 \mathrm{~dB}$ \}, which were shown in Figs. 7 and 8. Furthermore, 751 we applied our new adaption mechanism to the LTE turbo- 752 coded HARQ as a benchmarker for comparison. To exploit 753 the advantages of adaptive HARQ schemes, the fixed-rate LTE 754 turbo HARQ and our fixed-rate IRaptor HARQ are also charac- 755 terized in Figs. 7 and 8.

756

For all HARQ schemes, the ARQ-retry limit was set to 4. A 757 sufficiently high number of packets having an original informa- 758 tion length of 104 bits were BPSK modulated and transmitted 759 over a quasi-static, i.e., block-fading Rayleigh channel. In the 760 implementation of adaptive HARQ schemes, the real value of 761 each estimated SNR was rounded down to the closest integer for 762 determining the coding rate using our LUT. For example, an es- 763 timated SNR of $0.65 \mathrm{~dB}$ was rounded down to the SNR of $0 \mathrm{~dB} .764$

The LTE HARQ scheme is based on the turbo codes de- 765 scribed in Section III-B. In our simulations, the fixed-rate LTE 766 


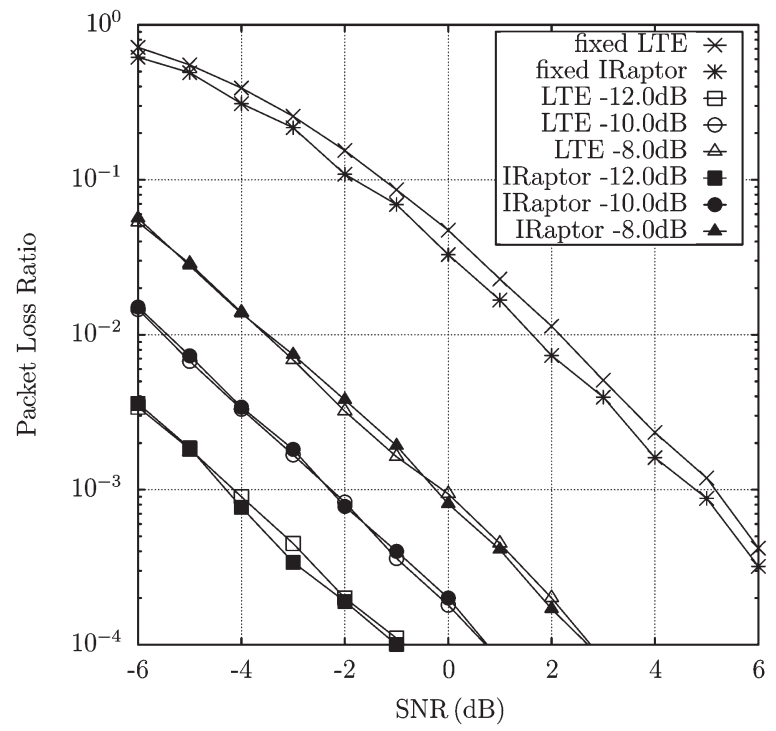

Fig. 7. PLR versus SNR for all candidate HARQ schemes for transmission over a quasi-static Rayleigh fading channel.

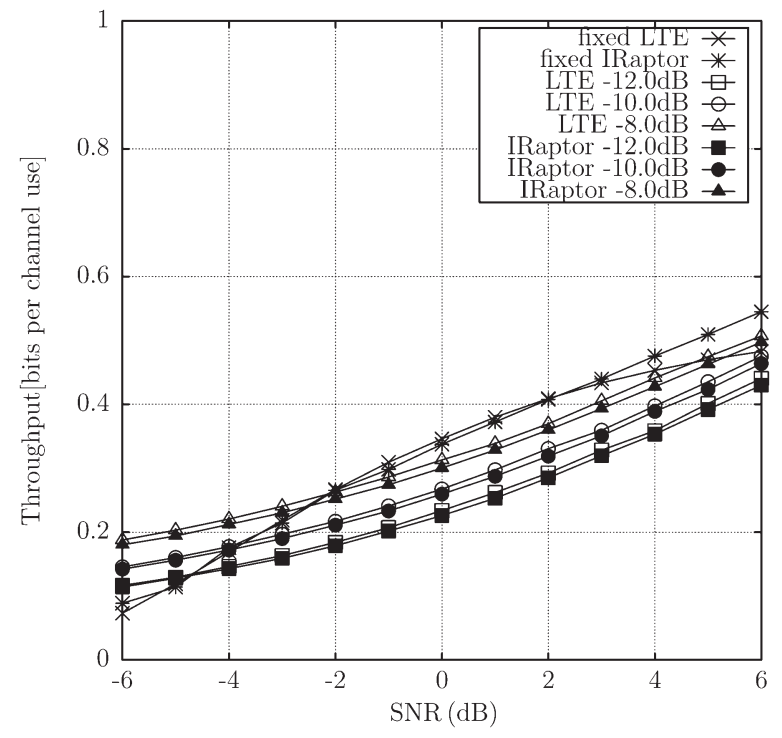

Fig. 8. Throughput versus SNR for all candidate HARQ schemes for transmission over a quasi-static Rayleigh fading channel.

767 HARQ employs the coding rate of 1 for each (re)transmission. 768 More specifically, the transmitter transmits 104 bits for each 769 of its four (re)transmissions, which are sequentially provided 770 by the circular buffer by advancing its starting position. At 771 the receiver, Chase combining is carried out for the repeated 772 LLRs received from all (re)transmissions. When our adaptive 773 mechanism is applied to the LTE HARQ, the coding rates in 774 the LUT of Table II are reused for different channel qualities 775 for the sake of fair comparison. The number of transmitted bits 776 corresponding to the coding rate may be obtained by circularly 777 forwarding the starting position along the circular buffer. This 778 circular operation aids the turbo-coded HARQ easily satisfy 779 any arbitrary coding rates. Therefore, Chase combining is po780 tentially required for the repetitions in a single transmission 781 if this transmission requires the coding rate less than $1 / 3$, which implies the starting position has been advanced along the 782 circular buffer more than one circle. In both the fixed-rate and 783 the adaptive LTE HARQ, iterative decoding will be executed 784 between two Bahl, Cocke, Jelinek, and Raviv (BCJR) decoders 785 until the CRC is satisfied or the MI is converged.

Observe from Fig. 8 that, although the fixed-rate IRaptor- 787 coded HARQ has a similar throughput to that of the LTE sys- 788 tem's fixed-rate turbo coded HARQ scheme, its PLR is slightly 789 lower, as shown in Fig. 7. Alternatively, we can say that it re- 790 quires an approximately $0.5 \mathrm{~dB}$ lower SNR for maintaining the 791 same PLR. However, Fig. 7 demonstrated that a significantly 792 lower PLR may be achieved by the adaptive HARQ arrange- 793 ment right across the entire SNR range, regardless of whether 794 our adaptive IRaptor HARQ or the LTE adaptive HARQ is 795 used, explicitly around 5-dB lower power may be used than 796 those of the nonadaptive HARQ, even for the highest threshold 797 of $\sigma_{\mathrm{th}}=-8 \mathrm{~dB}$. More specifically, when the threshold SNR 798 $\sigma_{\text {th }}$ is set for example to $-12 \mathrm{~dB}$, the PLR is below $10^{-3} 799$ for SNRs in excess of $-4 \mathrm{~dB}$. The PLR performance curve is 800 shifted to the right by about $2 \mathrm{~dB}$ for the threshold SNR $\sigma_{\text {th }}$ of 801 $-10 \mathrm{~dB}$, whereas the throughput observed in Fig. 8 exhibits an 802 improvement of about $4 \%$.

803

Note that the LTE adaptive HARQ scheme can be seen to 804 offer a similar PLR as our IRaptor HARQ scheme in Fig. 7. 805 Furthermore, Fig. 8 shows that both schemes facilitate similar 806 transmission throughputs. However, our IRaptor scheme has a 807 significant advantage over the LTE adaptive HARQ scheme in 808 terms of processing throughput and latency. More specifically, 809 turbo decoders rely on the BCJR algorithm, which employs 810 recursive calculations up and down the length of the trellis 811 [27]. These forward and backward recursions lead to lots of 812 data dependence, which prevent very parallel processing in 813 the turbo decoder hardware. In state-of-the-art turbo decoder 814 implementations [28], only 64 trellis stages can be processed 815 in each hardware clock cycle, resulting in an iterative turbo 816 decoding process that takes hundreds or thousands of clock 817 cycles to complete, depending on the frame length. By contrast, 818 the decoder of our IRaptor scheme is based upon a factor graph, 819 in which there are significantly fewer data dependencies. This 820 allows all of the VNs to be processed simultaneously in parallel, 821 and then all of the CNs to be processed simultaneously, in 822 parallel [29], [30]. Owing to this, the iterative decoding process 823 can be completed in only tens of clock cycles if a sufficiently 824 parallel processing architecture is employed (at the cost of 825 having a large chip area). Indeed, in [28], [31], and [32], it 826 is shown that LDPC decoders based on factor graphs can be 827 implemented with processing throughputs of tens of gigabits 828 per second, whereas the fastest BCJR-based turbo decoders 829 have throughputs of only 1 or $2 \mathrm{Gbit} / \mathrm{s}$. In summary, our IRaptor 830 scheme can be expected to facilitate an order-of-magnitude 831 improvement to the processing throughput and latency of the 832 LTE adaptive HARQ scheme, while maintaining the same PLR 833 and transmission throughput.

$$
834
$$

\section{CONCLUSION}

The family of rateless Fountain codes has been conceived for 836 filling packet erasures for transmission over BECs. However, 837 
838 owing to their suboptimum Tanner graph structure, their per839 formance erodes when they act as channel codes in the physical 840 layer of noise-contaminated fading channels. To circumvent 841 this impediment, we conceived a beneficial technique for incor842 porating several specifically encoded bits, which are generated 843 for eliminating the structural imperfections of their Tanner 844 graph. The resultant IRaptor codes exhibit an excellent PLR 845 performance, despite their low complexity.

846 Furthermore, a powerful IRaptor-coded LUT-aided HARQ 847 scheme was proposed for block-fading channels, where the 848 most appropriate code rate to be used was determined using 849 a LUT based on the estimated SNR. When considering our 850 rateless-coded HARQ scheme, the potentially infinite number 851 of coding rates is reduced to a finite number stored in our LUT. 852 In our future work, we may embark on tackling the challenge of 853 finding the most appropriate Tanner graph connection pattern 854 corresponding to each specific coding rate for various packet 855 lengths.

901 [18] X. Liu and T. J. Lim, "Fountain codes over fading relay channels," IEEE

\section{REFERENCES}

1] D. J. C. MacKay, "Fountain codes," Proc. Inst. Elect. Eng.-Commun., vol. 152, no. 6, pp. 1062-1068, Dec. 2005.

2] M. Luby, "LT codes," in Proc. 43rd Annu. IEEE Symp. Found. Comput. Sci., 2002, pp. 271-280.

3] A. Shokrollahi, "Raptor codes," IEEE Trans. Inf. Theory, vol. 52, no. 5, pp. 2033-2051, May 2006.

4] U. Erez, M. Trott, and G. Wornell, "Rateless coding for Gaussian channels," IEEE Trans. Inf. Theory, vol. 58, no. 2, pp. 530-547, Feb. 2012.

5] T.-Y. Chen, D. Divsalar, J. Wang, and R. Wesel, "Protograph-based raptorlike LDPC codes for rate compatibility with short blocklengths," in Proc. IEEE GLOBECOM Conf., 2011, pp. 1-6.

[6] R. Palanki and J. Yedidia, "Rateless codes on noisy channels," in Proc. ISIT, Jun. 2004.

[7] J. Castura and Y. Mao, "Rateless coding over fading channels," IEEE Commun. Lett., vol. 10, no. 1, pp. 46-48, Jan. 2006.

8] O. Etesami and A. Shokrollahi, "Raptor codes on binary memoryless symmetric channels," IEEE Trans. Inf. Theory, vol. 52, no. 5, pp. 2033-2051, May 2006.

9] K. Hu, J. Castura, and Y. Mao, "Performance-complexity tradeoffs of Raptor codes over Gaussian channels," IEEE Commun. Lett., vol. 11, no. 4, pp. 343-345, Apr. 2007.

0] B. Sivasubramanian and H. Leib, "Fixed-rate Raptor codes over Rician fading channels," IEEE Trans. Veh. Technol., vol. 57, no. 6, pp. 3905-3911, Nov. 2008.

1] Z. Cheng, J. Castura, and Y. Mao, "On the design of Raptor codes for binary-input Gaussian channels," IEEE Trans. Commun., vol. 57, no. 11, pp. 3269-3277, Nov. 2009.

2] K. Kasai and K. Sakaniwa, "Fountain codes with multiplicatively repeated non-binary LDPC codes," in Proc. 6th ISTC Iterative Inf. Process., 2010, pp. 374-378.

3] R. Tanner, "A recursive approach to low complexity codes," IEEE Trans. Inf. Theory, vol. 27, no. 5, pp. 533-547, Sep. 1981.

vol. 12 , no. 9 , pp. $681-683$, Sep. 2008

15] "3rd Generation Partnership Project; Technical Specification group radio access network; Evolved Universal Terrestrial Radio Access (E-UTRA); Multiplexing and channel coding (Release 10)," 3GPP TS 36.212, Sophia Antipolis Cedex, France, Dec. 2010. [Online]. Available: http://www.3gpp.org/FTP/Specs/html-info/26346.htm

16] F. Kschischang, B. Frey, and H.-A. Loeliger, "Factor graphs and the sumproduct algorithm," IEEE Trans. Inf. Theory, vol. 47, no. 2, pp. 498-519, Feb. 2001.

7] H. Jenkač and T. Mayer, "Soft decoding of LT-codes for wireless broadcast," in Proc. IST Mobile Summit, Jun. 2005.

Trans. Wireless Commun., vol. 8, no. 6, pp. 3278-3287, Jun. 2009

9] N. Bonello, R. Zhang, S. Chen, and L. Hanzo, "Reconfigurable rateless codes," IEEE Trans. Wireless Commun., vol. 8, no. 11, pp. 5592-5600, Nov. 2009.
[20] J. Hagenauer, E. Offer, and L. Papke, "Iterative decoding of binary 906 block and convolutional codes," IEEE Trans. Inf. Theory, vol. 42, no. 2, 907 pp. 429-445, Mar. 1996.

[21] S. Tong, P. Wang, D. Wang, and X. Wang, "Box-minus operation and 909 application in sum-product algorithm," Electron. Lett., vol. 41, no. 4, 910 pp. 197-198, Feb. 2005.

[22] H. Chen, R. G. Maunder, and L. Hanzo, "Low-complexity multiple- 912 component turbo decoding aided hybrid ARQ," IEEE Trans. Veh. 913 Technol., vol. 60, no. 4, pp. 1571-1577, May 2011.

914

[23] J. Hagenauer, "The EXIT chart-Introduction to extrinsic informa- 915 tion transfer in iterative processing," in Proc. 12th EUSIPCO, 2004, 916 pp. 1541-1548.

[24] X. Zuo, R. Maunder, and L. Hanzo, "Design of fixed-point process- 918 ing based LDPC codes using EXIT charts," in Proc. IEEE VTC-Fall, 919 Sep. 2011, pp. 1-5.

[25] L. Hanzo, T. Liew, B. Yeap, R. Tee, and S. Ng, Turbo Coding, Turbo 921 Equalisation and Space-Time Coding: EXIT-Chart-Aided Near-Capacity 922 Designs for Wireless Channels, 3rd ed. Hoboken, NJ, USA: Wiley, 923 2011.

[26] E. Soijanin, N. Varnica, and P. Whiting, "Punctured vs rateless codes 925 for hybrid ARQ," in Proc. IEEE ITW Punta del Este., Mar. 2006, 926 pp. $155-159$.

[27] L. Hanzo, J. P. Woodard, and P. Robertson, "Turbo decoding and de- 928 tection for wireless applications," Proc. IEEE, vol. 95, no. 6, pp. 1178- 929 1200, Jun. 2007.

930

[28] T. Ilnseher, F. Kienle, C. Weis, and N. Wehn, "A 2.15 Gbit/s turbo code 931 decoder for LTE advanced base station applications," in Proc. Int. Symp. 932 Turbo Codes Iterative Inf. Process., Gothenburg, Sweden, Aug. 2012, 933 vol. 1, pp. 21-25.

934

[29] C. Howland and A. Blanksby, "Parallel decoding architectures for low 935 density parity check codes," in Proc. IEEE ISCAS, May 2001, vol. 4, 936 pp. 742-745.

[30] F. Verdier and D. Declercq, "A low-cost parallel scalable FPGA architec- 938 ture for regular and irregular LDPC decoding," IEEE Trans. Commun., 939 vol. 54, no. 7, pp. 1215-1223, Jul. 2006.

[31] N. Onizawa, T. Hanyu, and V. Gaudet, "Design of high-throughput fully 941 parallel LDPC decoders based on wire partitioning," IEEE Trans. VLSI 942 Syst., vol. 18, no. 3, pp. 482-489, Mar. 2010.

[32] V. Chandrasetty and S. Aziz, "FPGA implementation of a LDPC decoder 944 using a reduced complexity message passing algorithm," J. Netw., vol. 6, 945 no. 1, pp. 36-45, Jan. 2011.

946

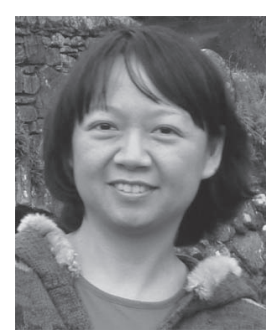

Hong Chen received the Master's degree in computer 947 science from the University of Electronic Science 948 and Technology (UESTC), Chengdu, China, and the 949 $\mathrm{Ph} . \mathrm{D}$. degree from the Communications Research 950 Group, School of Electronics and Computer Science, 951 University of Southampton, Southampton, U.K. 952

Since 2000, she has been a Lecturer with UESTC. 953 Upon the completion of her doctoral studies, she 954 became a Postdoctoral Research Fellow with the 955 University of Surrey, Guildford, U.K. Her current 956 research interests include cross-layer optimization of 957 wireless networks and turbo-coding-aided hybrid automatic repeat request. $\quad 958$

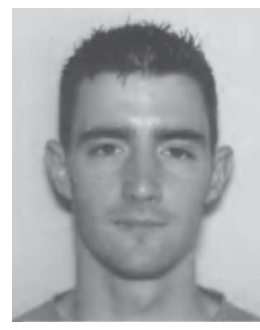

Robert G. Maunder (M'03) received the B.Eng. de- 959 gree in electronic engineering and the Ph.D. degree 960 in wireless communications from the University of 961 Southampton, Southampton, U.K., in 2003 and 2007, 962 respectively.

963

$\mathrm{He}$ is currently with the School of Electronics 964 and Computer Science, University of Southampton, 965 Southampton, U.K. He is the author of a number of 966 IEEE papers in these areas. His research interests 967 include video coding, joint source/channel coding, 968 and iterative decoding. 


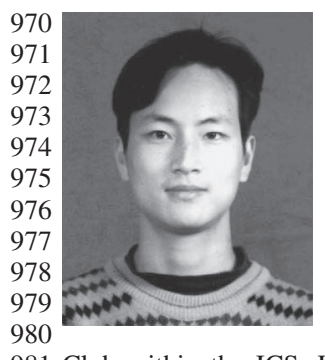

Yi Ma (M'04-SM'10) received the Ph.D. degree in electrical engineering and electronics from The University of Liverpool, Liverpool, U.K., in 2004

Since 2004, he has been with the Institute for Communication Systems (ICS), University of Surrey, Guildford, U.K., where he is currently a member of the academic staff. He is leading a research group within the ICS to conduct the basic-level communication systems research, covering signal processing and applied information theory for wireless communications. He is the Chair of the Air Interface 981 Club within the ICS. He is the author or coauthor of more than 100 peer982 reviewed IEEE journal and conference papers in the areas of cooperative 983 communications, cognitive radios, interference utilization, cooperative local984 ization, radio resource allocation, multiple-input multiple-output, estimation, 985 synchronization, modulation, and detection techniques. He has three patents in 986 the areas of spectrum sensing, signal modulation, and detection. He is currently 987 the Technical Coordinator of FP7 ICT-RESCUE project.

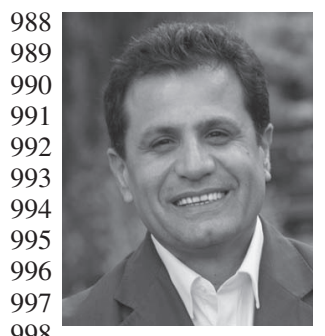

\section{Rahim Tafazolli (SM'09)}

$\mathrm{He}$ is currently the Director of the Institute for Communication Systems and of the 5G Innovation Centre, University of Surrey, Guildford, U.K. He is the author of more than 500 research papers in refereed journals and international conferences and has served as an invited speaker. He currently has more than 20 patents in the field of mobile communications. He is the editor of two books on Technologies for Wireless Future. He has also worked as a consultant to many mobile companies. He brings 999 his international standing in the communications technology academic and 1000 industrial community and his expert technical knowledge of the field to the 1001 project.

1002 Mr. Tafazolli is a Fellow of The Institution of Engineering and Technology 1003 and Wireless World Research Forum. He has lectured, chaired, and been invited 1004 as a keynote speaker to a number of IET and IEEE workshops and conferences. 1005 He currently serves as the Chair for the EU Net!Works Technology Platform 1006 Expert Group, as a board member of the U.K. Future Internet Strategy Group, 1007 and as a Coordinator of the IoT-i Coordination Action Project.

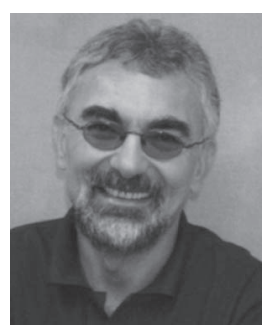

Lajos Hanzo (F'04) received the Master's degree 1008 in electronics, the Ph.D. degree, and the Doctor 1009 Honoris Causa degree from the Technical University 1010 of Budapest, Budapest, Hungary, in 1976, 1983, 1011 and 2009 respectively, and the D.Sc. degree from 1012 the University of Southampton, Southampton, U.K., 1013 in 2004.

1014

During his career in telecommunications, he 1015 has held various research and academic posts in 1016 Hungary, Germany, and the U.K. Since 1986, he has 1017 been with the School of Electronics and Computer 1018 Science, University of Southampton, Southampton, U.K., where he holds 1019 the Chair in telecommunications. He was a Chaired Professor of Tsinghua 1020 University, Beijing, China. He is the coauthor of 20 John Wiley/IEEE Press 1021 books on mobile radio communications, totalling in excess of 10000 pages, and 1022 has published more than 1400 research entries on IEEE Xplore. He is currently 1023 directing an academic research team, working on a range of research projects 1024 in the field of wireless multimedia communications sponsored by industry, 1025 the Engineering and Physical Sciences Research Council (EPSRC) U.K., the 1026 European IST Program, and the Mobile Virtual Centre of Excellence, U.K. He 1027 is an enthusiastic supporter of industrial and academic liaison, and he offers a 1028 wide range of industrial courses.

Dr. Hanzo has acted as a Technical Program Committee Chair for IEEE con- 1030 ferences, presented keynote lectures, and has received a number of distinctions. 1031 $\mathrm{He}$ is the Governor of the IEEE Vehicular Technology Society and the Past 1032 Editor-in-Chief of the IEEE Press. 


\section{AUTHOR QUERIES}

\section{AUTHOR PLEASE ANSWER ALL QUERIES}

AQ1 = Please provide keywords

AQ2 = Please provide page range and location for Ref. [6].

AQ3 = Please provide page range and location for Ref. [17].

AQ4 = Kindly provide the academic history of R. Tafazolli.

END OF ALL QUERIES 